\title{
Sleep bolsters schematically incongruent memories
}

Jennifer E. Ashton ${ }^{1}$, Bernhard P. Staresina ${ }^{2} \&$ Scott A. Cairney ${ }^{1,3^{*}}$

\footnotetext{
${ }^{1}$ Department of Psychology, University of York, York, UK

${ }^{2}$ Department of Experimental Psychology, University of Oxford, Oxford, UK

${ }^{3}$ York Biomedical Research Institute, University of York, York, UK

* Corresponding author (scott.cairney@york.ac.uk)
} 


\section{Abstract}

Our ability to recall memories is improved when sleep follows learning, suggesting that sleep facilitates memory consolidation. A number of factors are thought to influence the impact of sleep on newly learned information, such as whether or not we rehearse that information (e.g. via restudy or retrieval practice), or the extent to which the information is consistent with our pre-existing schematic knowledge. In this pre-registered, online study, we examined the effects of both rehearsal and schematic congruency on overnight consolidation. Participants learned noun-colour pairings (e.g. elephant-red) and rated each pairing as plausible or implausible before completing a baseline memory assessment. Afterwards, participants engaged in a period of restudy or retrieval practice for the pairings, and then entered a $12 \mathrm{~h}$ retention interval of overnight sleep or daytime wakefulness. Follow-up assessments were completed immediately after sleep or wake, and again $24 \mathrm{~h}$ after learning. Our data indicated that overnight consolidation was amplified for restudied relative to retested noun-colour pairings, but only when sleep occurred soon after learning. Furthermore, whereas plausible (i.e. schematically congruent) pairings were generally better remembered than implausible (i.e. schematically incongruent) pairings, sleep (vs wake) reduced the retention advantage for plausible (vs implausible) information. This finding challenges the notion that schema-conformant memories are preferentially strengthened during post-learning sleep. 


\section{Introduction}

Memories fade over time, but rehearsing learned materials can help to improve recall. There are two strategies that an individual can use to help commit new information to memory: restudy and retrieval practice. For example, one might try to revise for a spelling test by rereading the words (restudy) or by attempting to recall the words from memory (retrieval practice). In the short term (e.g. within an hour of learning), restudied information is better remembered than information subjected to retrieval practice. In the longer-term (e.g. from several hours up to week), by contrast, the benefits of retrieval practice tend to outweigh those arising from restudy (Carpenter et al., 2008; Roediger \& Karpicke, 2006). This difference in retention after longer intervals suggests that restudied memories might be more susceptible to decay than those subjected to retrieval practice.

Memory retention is improved by post-learning sleep (Ashton et al., 2020; Ashton \& Cairney, 2021; Cairney, Lindsay, Paller, \& Gaskell, 2018; Durrant, Cairney, \& Lewis, 2016; Gaskell, Cairney, \& Rodd, 2019; Rasch \& Born, 2013), suggesting that sleep supports the consolidation of newly learned information. Contemporary models of sleep-associated memory processing propose that memories are reactivated during sleep, and thereby integrated into long-term storage (Antony et al., 2019; Diekelmann \& Born, 2010; Klinzing, Niethard, \& Born, 2019; Lewis \& Durrant, 2011; Rasch \& Born, 2007, 2013). However, sleep does not benefit all memories equally, with accumulating evidence suggesting that overnight memory gains are more robust for weakly encoded than strongly encoded materials (Denis et al., 2021, 2020; Payne, Chambers, \& Kensinger, 2012; Schmidt et al., 2006, but also see Petzka et al., 2021). Relatedly, efforts to enhance overnight consolidation via memory cueing in sleep are most effective when pre-sleep learning performance is low (Cairney, Lindsay, Sobczak, Paller, \& Gaskell, 2016; Creery, Oudiette, Antony, \& Paller, 2015; Schechtman et al., 2021).

Given that sleep may provide the greatest benefit to weakly encoded memories, and that restudied memories are more prone to decay than memories subjected to retrieval practice, restudied information should be particularly responsive to overnight consolidation. Consistent with this view, previous work has indicated that restudied but not retested materials are better remembered after a night of sleep than a day of wakefulness (Antony \& Paller, 2018; Bäuml, Holterman, \& Abel, 2014). It has thus been suggested that retrieval practice may prompt a rapid consolidation of newly learned information into long-term memory, potentially via similar mechanisms to those underpinning sleep-associated memory processing (Antony et al., 2017).

Information that is congruent with pre-existing schematic knowledge is typically better remembered than non-congruent information (Sommer, 2017; Tse et al., 2007, 2011). The memory benefits of cognitive schemata are thought to arise from interactions between prefrontal cortex and medial temporal lobe, which support efficient learning of schematically 
congruent materials (van Kesteren et al., 2012; 2013). Interestingly, prior knowledge also enhances sleep-associated consolidation, such that schema-conformant memories are strengthened during sleep to a greater extent than non-conformant memories (Durrant, Cairney, McDermott, \& Lewis, 2015; Groch et al., 2017; Wamsley et al., 2010). The interleaved reactivation of new memories and their associated schematic representations during sleep is thought to facilitate the integration of newly learned information into long-term storage (Lewis \& Durrant, 2011).

In this pre-registered, online replication study (osf.io/f82mw), we tested two hypotheses: 1 ) the benefits of sleep (vs wakefulness) for memory will emerge for restudied memories but not memories subjected to retrieval practice, and 2) the retention advantage for schematically congruent (vs incongruent) memories will be stronger after sleep than wakefulness. We also tested a third novel hypothesis concerning the combined effects of sleep, memory rehearsal and prior knowledge on memory consolidation. Assuming that overnight memory processing preferentially strengthens restudied (vs retested) and schematically congruent (vs incongruent) memories, then, after sleep (vs wake), the benefits of restudy (vs retrieval practice) should be greater for schema-conformant than nonconformant information.

We tested our three hypotheses using a source memory paradigm in which participants learned noun-colour pairings (e.g. elephant-red) and rated the plausibility of each pairing (e.g. a red elephant is implausible). From this plausibility response, we could infer the schematic congruency of each pairing, which was based on the participant's unique understanding of the world. Training (encoding and baseline memory assessment) took place in the morning or evening and was immediately followed by a memory rehearsal phase, during which participants engaged in a period of restudy or retrieval practice for the pairings. Memory for the pairings was re-assessed $12 \mathrm{~h}$ later, following a night of sleep (evening training) or a day of wakefulness (morning training). This allowed us to determine the effects of sleep (vs wake) on the consolidation of memories that had been restudied or retested, and were plausible (i.e. schematically congruent) or implausible (i.e. schematically incongruent). Finally, so that we could also determine the effects of sleep on memory retention after a longer delay, participants completed a second follow-up assessment $24 \mathrm{~h}$ after training.

\section{Methods}

\section{Participants}

One-hundred and sixty-eight adults were recruited via the online platform Prolific (app.prolific.co). Participants were aged between 18 and 30 years and reported to be living in the UK with English as their first language. On the days of the study, participants were asked to follow their usual daily routines, abstain from alcohol and avoid taking naps. Informed 
consent was obtained from all participants in line with the Research Ethics Committee of the Department of Psychology, University of York, who approved the study. Participants were required to meet a memory performance criterion in the first session (see below) to continue with the remaining sessions. A total of 102 participants met this criterion and were invited to take part in sessions two and three. Sixty-two participants returned to complete the full study. We analysed data from a final sample of 60 participants after excluding two participants who reported napping during the days of the experiment. The remaining participants took part in a sleep ( $n=30,18$ females, mean \pm SD age $=25.07 \pm 4.02$ years) or wake group $(n=30,25$ females, mean \pm SD age $=25.00 \pm 3.65$ years) .

Our sample size was calculated using an effect size reported in Antony \& Paller (2018). The effect of interest was an interaction $\left(\eta_{p}{ }^{2}=0.14\right)$ from a two-way ANOVA with the factors Delay (AM/PM) and rehearsal strategy (Restudy/Retest). We determined that a minimum sample size of $N=20$ would be necessary for $95 \%$ power to detect an effect of this magnitude. However, because Antony \& Paller (2018) conducted their study in the laboratory, we believed it reasonable to increase our sample to $N=60$ to mitigate any noise associated with online data collection. Data collection continued until our desired sample size was met, with each participant providing a full and useable data set.

\section{Materials and Software}

Six-hundred and eighty English nouns referring to concrete objects were obtained from the Medical Research Council Psycholinguistics database (websites.psychology.uwa.edu.au/school/mrcdatabase/uwa_mrc.htm). Words were three to eight letters long, with a Kucera-Francis written frequency of 10-100. Only words with concreteness and imageability ratings ranging from 400 to 700 (out of 700 ) were included (Staresina \& Davachi, 2008). For each participant, 240 nouns were randomly selected for the encoding phase and paired with one of four colours (red, yellow, green or blue). An equal number of nouns were paired with each colour (i.e. 60 nouns per colour). The remaining nouns were randomly assigned to each of the three test phases as foils (120 in each test). The experimental tasks were programmed using PsychoPy and hosted on Pavlovia.org (Peirce et al., 2019). Participants completed the tasks at home on a desktop or laptop (the use of tablets or smartphones was prohibited).

\section{Procedure Overview}

The study was divided into three sessions (see Figure 1A). Session one began at 8am (wake group) or 8pm (sleep group), and was composed of an encoding phase, a baseline memory test and a memory rehearsal phase. Follow-up memory tests were completed at sessions two and three, which began $12 \mathrm{~h}$ and $24 \mathrm{~h}$ after session one, respectively. The Stanford Sleepiness Scale (Hoddes et al., 1973) and a three-minute psychomotor vigilance task (Ashton, Jefferies, \& Gaskell, 2018; Khitrov et al., 2014) were completed at each session to measure participant alertness. 


\section{A. Procedure}

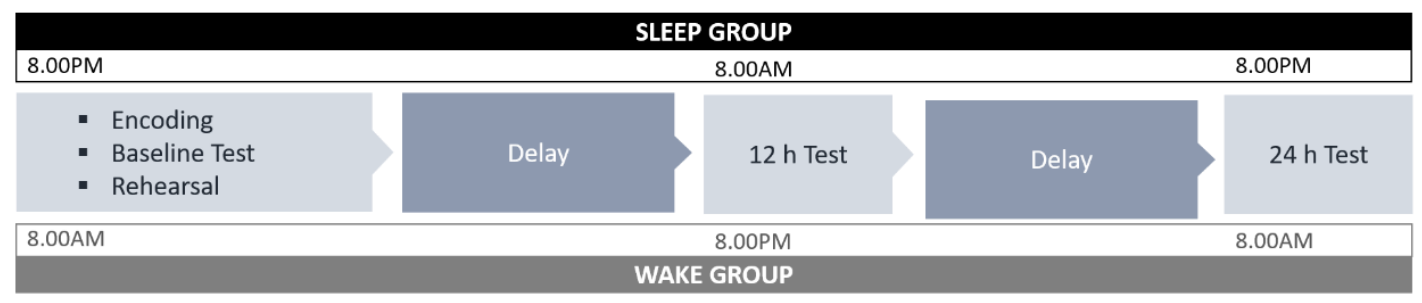

\section{B. Encoding}

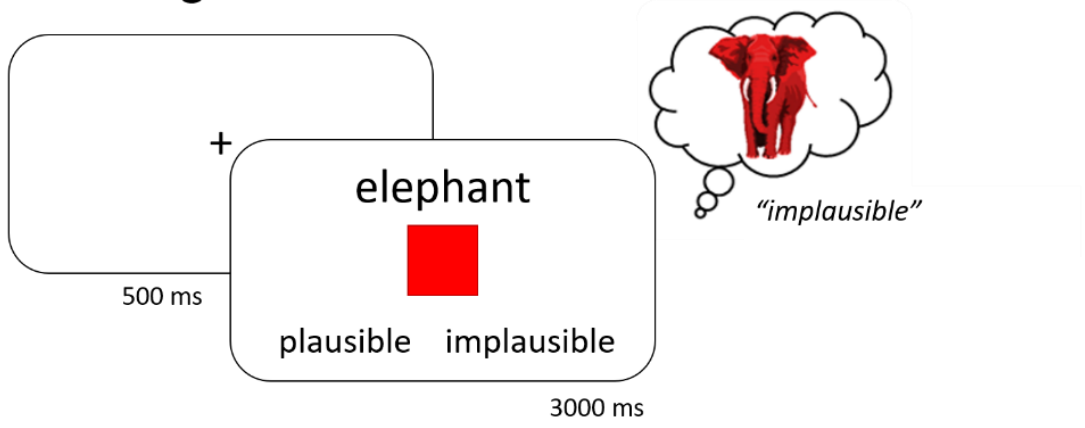

\section{Test}

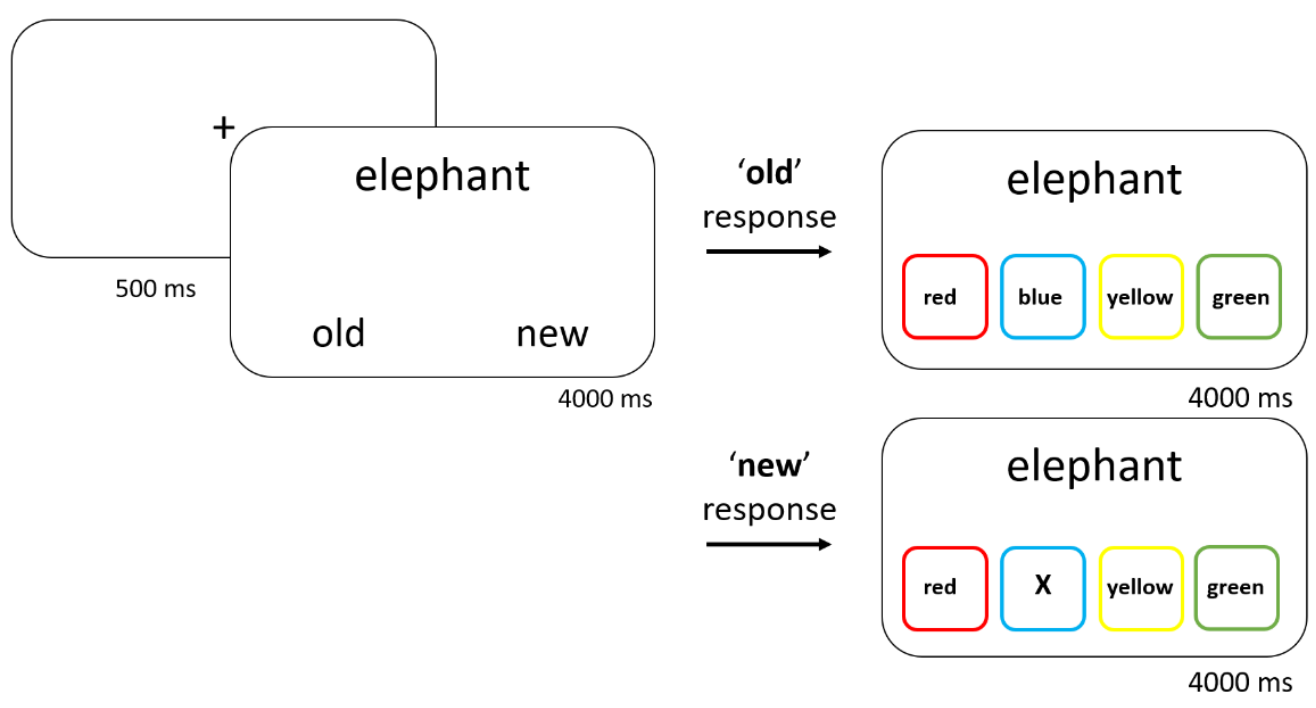

Figure 1. Experimental procedure and tasks. (A) Participants completed an encoding phase, a baseline memory test and a memory rehearsal phase in the morning (wake group) or evening (sleep group). Participants returned $12 \mathrm{~h}$ and $24 \mathrm{~h}$ later to complete follow-up memory tests. (B) On each encoding trial, participants were presented with a noun and a coloured square. Participants were asked to imagine the referent of the noun in the given colour and indicate whether it was a plausible or implausible combination. (C) On each test trial, participants were asked to decide whether a noun was 'old' (i.e. they had seen the noun at encoding) or 'new' (i.e. they had not seen the noun at encoding). For 'old' responses, participants were asked to select the colour that had appeared with the noun at encoding. For 'new' responses participants were asked to indicate the location of the letter ' $X$ '.

Encoding: There were 240 encoding trials. Each trial began with a fixation cross, presented in the centre of the screen for $500 \mathrm{~ms}$. A randomly selected noun was then displayed above a coloured square for $3 \mathrm{~s}$ (see Figure 1B). Participants were asked to imagine the referent of the noun in the given colour (red, yellow, green or blue) and indicate whether it was a plausible 
or implausible combination (e.g. a red elephant is implausible). As indicated by a 2 (Group: Sleep/Wake) * 2 (Plausibility: Plausible/Implausible) mixed ANOVA, there were an equivalent number of plausible and implausible responses in both the sleep and wake groups (all $p>.05$ ). Any trial for which a participant failed to provide a plausible or implausible response was removed from all subsequent analyses. On average, $3.11 \%$ of trials (SEM \pm 0.64 ) were removed on this basis.

Baseline Test: There were 360 test trials; 240 of which corresponded to the nouns presented at encoding. The remaining 120 trials were unseen foils. On each trial, a central fixation cross (500 ms) preceded a randomly selected noun (see Figure 1C). Participants were asked to indicate whether the noun was 'old' (i.e. they recognised it from encoding) or 'new' (i.e. they did not recognise it from encoding). For each 'old' response, participants were also asked to select the colour that had been paired with the noun at encoding, and guess if they were not certain. For each 'new' response, participants were asked to indicate the location of a letter ' $X$ ' that was randomly presented in one of four locations. Employing this two-step approach provided an index of item memory (noun recognition) and source memory (colour recall). To motivate engagement with the task, participants were informed of their score at the end of the baseline test (calculated as the percentage of correct old/new responses) and encouraged to try and improve on it in the next test.

Performance Criteria: If a participant did not meet the following baseline performance criteria, they were not invited back for the follow-up tests and a new participant was recruited in their place. We excluded 66 participants on this basis.

The performance criteria were set in two steps:

1) Item memory score of $50 \%$ or above. Calculated as the proportion of correctly recognised 'old' nouns, minus the proportion of foils incorrectly rated as 'old'.

2) Source memory score of $40 \%$ or above. Calculated as the proportion of correctly identified colours (restricted to correctly recognised nouns), referred to hereafter as colour hits.

Memory Rehearsal: After the baseline test, participants completed two rehearsal phases: restudy and retrieval practice (order counterbalanced). The correctly recognised nouns for which participants had also correctly identified the associated colour were randomly and equally allocated to three conditions: restudy, retrieval practice and a no-rehearsal control condition. The restudy and retrieval practice phases followed the same procedures as at encoding and the baseline test, respectively.

Follow-up Tests: Participants completed a follow-up test $12 \mathrm{~h}$ after the baseline test (i.e. after a night of sleep [sleep group] or a day of wakefulness [wake group]). A second follow-up test 
took place $24 \mathrm{~h}$ after the baseline assessment. Both follow-up tests followed the same procedures as the baseline test, with the exception that a new set of foils were used in each.

\section{Statistical Analysis}

Our dependent variable of interest was source memory retention, calculated for the $12 \mathrm{~h}$ and $24 \mathrm{~h}$ follow-up tests as the number of colour hits divided by the number of colour hits at baseline. Data at each follow-up test was applied to a 2 (Group: Sleep/Wake) * 3 (Rehearsal Strategy: Restudy/Retrieval Practice/No-Rehearsal) * 2 (Plausibility: Plausible/Implausible) mixed ANOVA. The plausibility of each noun-colour pairing was determined by the plausible/implausible responses provided at encoding. Significant interactions were interrogated using post-hoc t-tests with a Bonferroni-corrected alpha adjustment for the number of tests performed. All statistical analyses were performed in R (R Core Team, 2019).

\section{Results}

\section{Baseline Performance}

Source memory scores (colour hits) at the baseline test (see Table 1) were applied to a 2 (Group: Sleep/Wake) * 2 (Plausibility: Plausible/Implausible) mixed ANOVA. Across both groups, plausible associations were better remembered than implausible associations $F(1,58)$ $\left.=127.22, p<.001, \eta_{p}^{2}=0.69\right)$. There was no significant difference in baseline performance between the sleep and wake groups $(F(1,58)=0.23, p=.630)$, and no Group * Plausibility interaction $(F(1,58)=0.06, p=.802)$.

\begin{tabular}{ccc}
\hline & Plausible & Implausible \\
\hline Sleep & $72.99 \pm 2.26$ & $60.85 \pm 2.13$ \\
Wake & $71.79 \pm 2.23$ & $59.10 \pm 2.55$ \\
\hline
\end{tabular}

Table 1. Source memory scores (colour hits) at the baseline test for the sleep and wake groups. Scores are presented separately for each plausibility condition. Data are presented as mean \pm SEM.

\section{Sleep and Rehearsal}

We first tested the hypothesis that a memory benefit of sleep would emerge for restudied source memories but not source memories subjected to retrieval practice.

\section{2 h Follow-up}

There was a main effect of Group $\left(F(1,58)=11.39, p=.001, \eta_{p}{ }^{2}=0.16\right)$, indicating that source memory retention was significantly higher after a night of sleep than a day of wakefulness (see Table 2 and Figure 2). There was also a main effect of Rehearsal Strategy $(F(2,116)=$ 76.53, $\left.p<.001, \eta_{p}^{2}=0.57\right)$ : retention scores were higher in 1$)$ the restudy vs no-rehearsal 
condition $(t(59)=12.58, p<.001, d=1.62), 2)$ the retrieval practice vs no-rehearsal condition $(t(59)=8.51, p<.001, d=1.10)$, and 3$)$ the restudy vs retrieval practice condition $(t(58)=2.90$, $p=.005, d=0.38)$, indicating that restudy produced the greatest overall retention benefit. However, there was no Group * Rehearsal Strategy interaction $(F(2,116)=1.70, p=.187)$, indicating that the memory effects of sleep were not amplified in any of the rehearsal conditions.

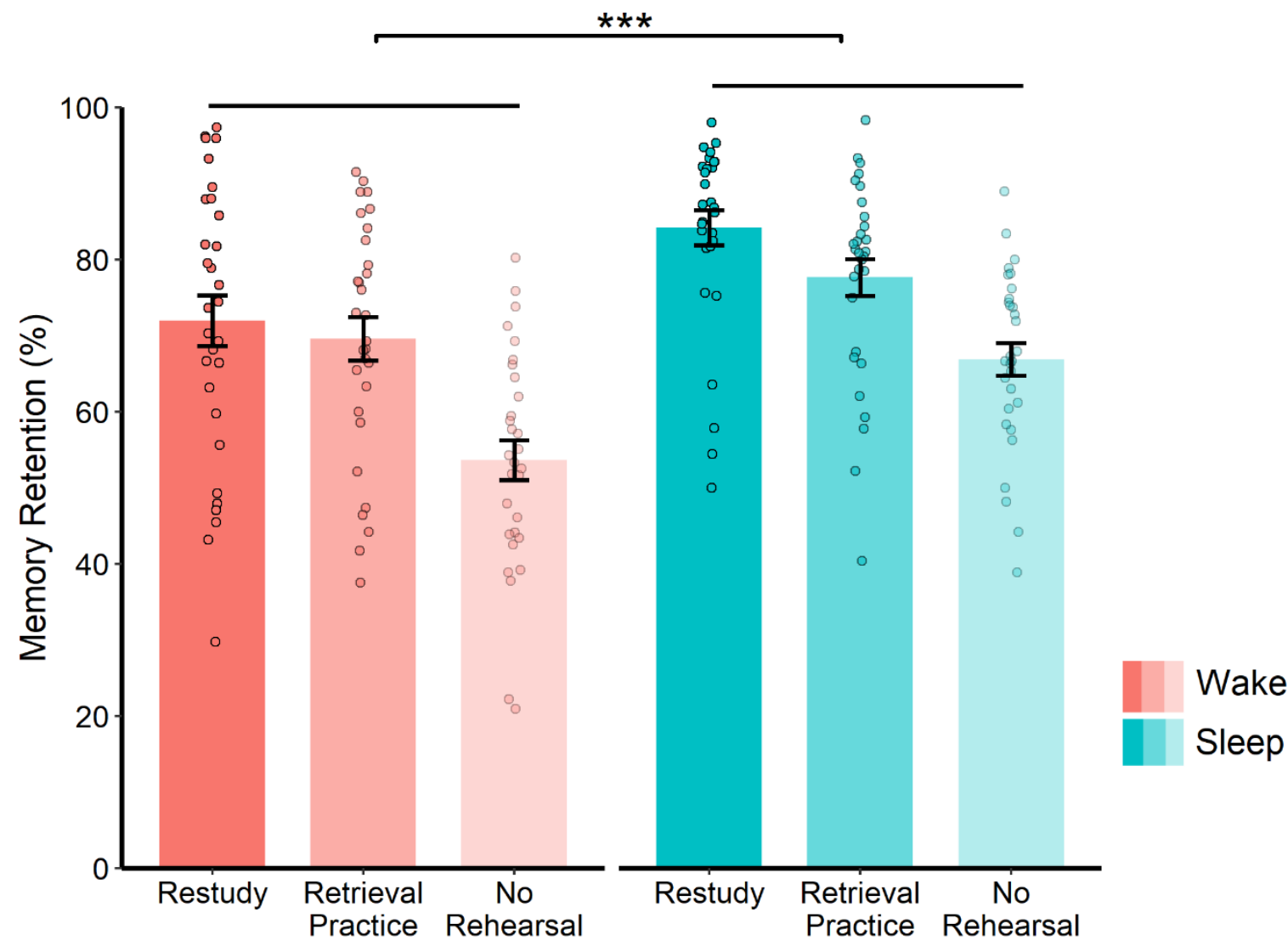

Figure 2. Source memory retention at the $12 \mathrm{~h}$ follow-up for the sleep and wake groups. Data is shown for each rehearsal condition, collapsed across the plausibility conditions. Error bars represent SEM; ${ }^{* *} p<.001$.

\section{$24 \mathrm{~h}$ Follow-up}

There was no main effect of Group at the $24 \mathrm{~h}$ follow-up $(F(1,58)=0.39, p=.535)$, but the main effect of Rehearsal Strategy remained $\left(F(2,116)=83.31, p<.001, \eta_{p}{ }^{2}=0.59\right)$ : retention scores were again higher in 1$)$ the restudy vs no-rehearsal condition $(t(58)=13.72, p<.001$, $d=1.77), 2$ ) the retrieval practice vs no-rehearsal condition $(t(58)=8.81, p<.001, d=1.14)$, and 3 ) the restudy vs retrieval practice condition $(t(58)=2.67, p=.010, d=0.35)$. Interestingly, a significant Group * Rehearsal Strategy interaction also emerged at the $24 \mathrm{~h}$ follow-up $\left(F(2,116)=3.63, p=.029, \eta_{p}^{2}=0.06\right.$; see Figure 3$)$. Whereas source memory retention was significantly higher after restudy than retrieval practice in the sleep group $(t(29)$ $=4.11, p<.001, d=.75)$, no such difference emerged in the wake group $(t(29)=0.49, p=$ .626). Hence, after a $24 \mathrm{~h}$ delay, the retention advantage for restudied (vs retested) information was only evident among individuals who had slept soon after learning. 


\begin{tabular}{lcccccc}
\hline & \multicolumn{2}{c}{ Restudy } & \multicolumn{2}{c}{ Retrieval Practice } & \multicolumn{2}{c}{ No-Rehearsal } \\
\multicolumn{2}{c}{ Plausible } & Implausible & Plausible & Implausible & Plausible & Implausible \\
\hline 12 h Follow-up & & & & & \\
Sleep $86.99 \pm 2.01$ & $81.35 \pm 3.12$ & $82.26 \pm 2.11$ & $73.05 \pm 3.15$ & $73.28 \pm 2.48$ & $60.55 \pm 2.46$ \\
Wake $\quad 77.49 \pm 3.04$ & $66.40 \pm 4.34$ & $75.11 \pm 2.51$ & $64.05 \pm 3.52$ & $62.93 \pm 2.78$ & $44.29 \pm 3.38$ \\
& & & & & & \\
24 h Follow-up & & & & & \\
Sleep $80.25 \pm 3.00$ & $74.56 \pm 3.72$ & $76.37 \pm 3.01$ & $64.66 \pm 3.71$ & $67.37 \pm 3.67$ & $54.38 \pm 3.32$ \\
Wake $79.41 \pm 2.83$ & $67.80 \pm 3.78$ & $76.74 \pm 2.60$ & $68.07 \pm 2.67$ & $63.46 \pm 2.84$ & $47.84 \pm 3.43$ \\
\hline
\end{tabular}

Table 2. Source memory scores (\%) for the $12 \mathrm{~h}$ and $24 \mathrm{~h}$ follow-ups in the sleep and wake groups. Scores are presented separately for each rehearsal strategy and plausibility condition. Data are presented as mean \pm SEM.

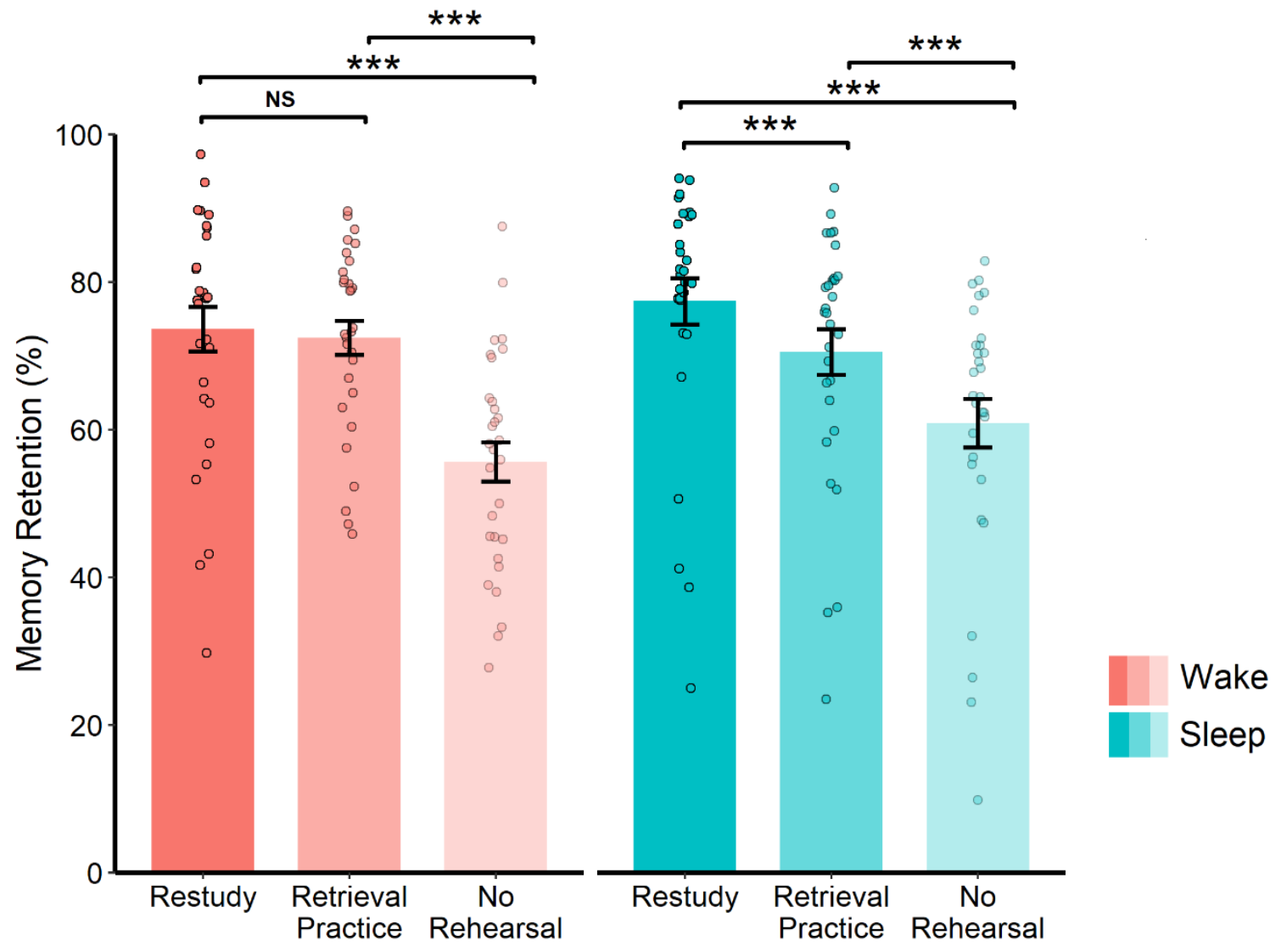

Figure 3. Source memory retention at the $24 \mathrm{~h}$ follow-up for the sleep and wake groups. Data is shown for each rehearsal condition, collapsed across the plausibility conditions. Error bars represent SEM; ${ }^{* * *} p<.001 ; \mathrm{NS}=$ not significant. 


\section{Sleep and Schematic Congruency}

Next, we tested the hypothesis that overnight consolidation strengthens schematically congruent (plausible) source memories to a greater extent than incongruent (implausible) source memories.

\section{2 h Follow-up}

There was a main effect of Plausibility $\left(F(1,58)=109.64, p<.001, \eta_{p}{ }^{2}=0.65\right)$, indicating that source memory retention was higher for plausible relative to implausible pairings. Importantly, there was also a significant Group * Plausibility interaction $(F(1,58)=4.10, p=$ $.048, \eta_{p}{ }^{2}=0.07$ ), but not in the direction predicted. The retention advantage for plausible (vs implausible) pairings was smaller in the sleep group $(t(29)=6.18, p<.001, d=1.13)$ than the wake group $(t(29)=8.56, p<.001, d=1.56$; see Figure $4 \mathrm{~A})$. Taken together, these findings suggest that sleep preferentially strengthened implausible associations.

\section{Follow-up}

The main effect of Plausibility was still present at the $24 \mathrm{~h}$ follow-up $(F(1,58)=87.36, p<.001$, $\left.\eta_{p}^{2}=0.60\right)$, but the Group * Plausibility interaction no longer remained $(F(1,58)=0.61, p=$ .439). However, under the assumption that sleep preferentially strengthens implausible associations, this null effect is to be expected, as participants in the wake group had also slept before the $24 \mathrm{~h}$ follow-up. To test this possibility directly, we carried out an exploratory analysis of source memory retention between the $12 \mathrm{~h}$ and $24 \mathrm{~h}$ follow-ups. If sleep selectively facilitated the consolidation of schematically incongruent associations, then the retention of implausible (vs plausible) pairings should be greater in the wake group than the sleep group, as only participants in the wake group had slept between these two time points.

Source memory retention scores were recalculated as the number of colour hits at the $24 \mathrm{~h}$ follow-up divided by the number of colour hits at the $12 \mathrm{~h}$ follow-up (a score above $100 \%$ indicates an improvement between the two assessments) and then reapplied to the ANOVA described above. Consistent with the memory benefits of sleep observed at the $12 \mathrm{~h}$ follow up, a main effect of Group emerged in this new ANOVA, with the wake group outperforming the sleep group at the $24 \mathrm{~h}$ follow-up $\left(F(1,58)=19.47, p<.001, \eta_{p}{ }^{2}=0.25\right)$. There was no main effect of Plausibility $(F(1,58)=3.39, p=.071)$.

Importantly, a significant Group * Plausibility interaction was also observed $(F 1,58)=8.23, p$ $=.006, \eta_{p}^{2}=0.12$; see Figure $4 B$ ). Whereas retention performance was higher for implausible relative to plausible associations in the wake group $(t(29)=2.84, p=.008, d=.52)$, no such difference emerged in the sleep group $(t(29)=0.92, p=.366)$. Taken together with the results of our main analysis, these findings suggest that overnight consolidation preferentially strengthens implausible associations, and, in doing so, closes the retention gap between plausible (i.e. schematically congruent) and implausible (i.e. schematically incongruent) information. 
A

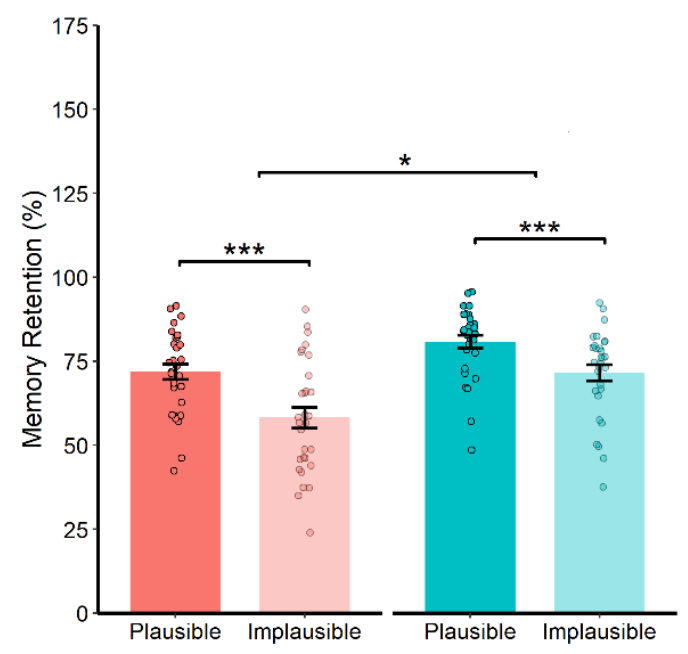

B

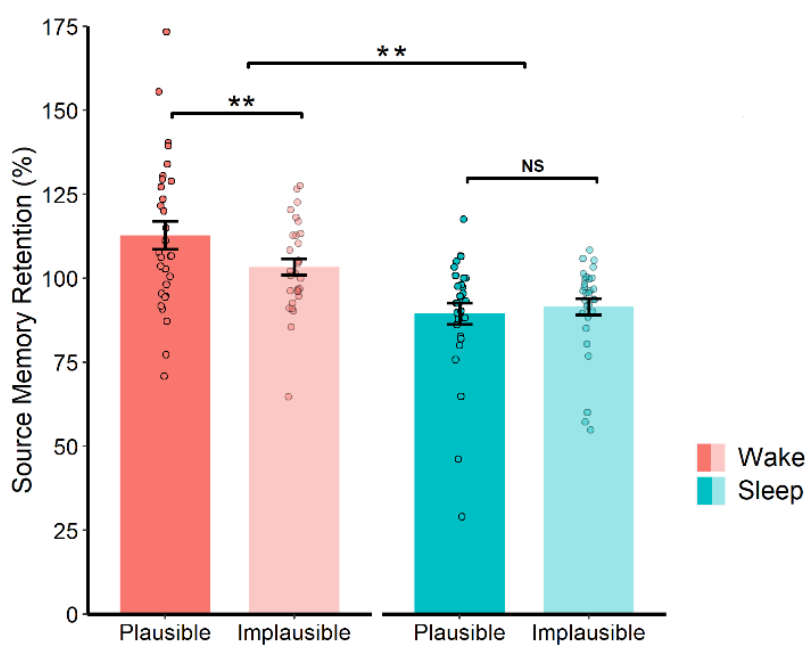

Figure 4. Memory retention at the (A) $12 \mathrm{~h}$ and (B) $24 \mathrm{~h}$ follow-ups. Note that retention scores at the $24 \mathrm{~h}$ followup are calculated as a proportion of retention at the $12 \mathrm{~h}$ follow-up (scores above $100 \%$ indicate an improvement between the two time points). Data are collapsed across the rehearsal conditions. Error bars represented SEM. $* * * p<.001, * * p<.01, * p<.05, \mathrm{NS}=$ non-significant.

\section{Sleep, Rehearsal and Schematic Congruency}

Finally, we tested the hypothesis that sleep-associated consolidation is enhanced for plausible (schematically congruent) memories that have been restudied prior to sleep. However, there was no significant Group * Rehearsal Strategy * Plausibility interaction at either the $12 \mathrm{~h}$ $(F(2,116)=0.31, p=.736)$ or $24 \mathrm{~h}$ follow-up $(F(2,116)=1.37, p=.258)$.

\section{Self-reported Sleep}

Participants reported the number of hours that they had slept between sessions one and two (Sleep group) or between sessions two and three (Wake group). Mean ( \pm SEM) hours slept in the Sleep group $(6.70 \pm 0.26)$ did not correlate with source memory retention scores in any of the Rehearsal Strategy/Plausibility conditions at the $12 \mathrm{~h}$ follow-up (all $p>.05$ ). Neither did the reported hours of sleep in the Wake group $(7.37 \pm 0.18$ ) at the $24 \mathrm{~h}$ follow-up (all $p>.05$ ).

\section{Alertness and Vigilance}

\section{Stanford Sleepiness Scale}

Scores on the Stanford Sleepiness Scale (SSS; see Table 3), were subjected to a 2 (Group: Sleep/Wake) * 3 (Session: Encoding/12 h/24 h) mixed ANOVA. There was a main effect of Session $\left(F(2,116)=5.04, p=.008, \eta_{p}^{2}=0.08\right)$, with participants rating themselves as more alert at encoding than at the $12 \mathrm{~h}$ follow-up $(t(59)=3.07, p=.003, d=0.40)$. All other between-session differences were non-significant (all $p>.05$ ). There was no main effect of $\operatorname{Group}(F(1,58)=2.65, p=.109)$ and no Group * Session interaction $(F(2,116)=1.04, p=.358)$. 


\begin{tabular}{lcccccc}
\hline & \multicolumn{2}{c}{ Encoding } & \multicolumn{2}{c}{ 12 h Follow-up } & \multicolumn{2}{c}{ 24 h Follow-up } \\
& SSS & PVT Lapse & SSS & PVT Lapse & SSS & PVT Lapse \\
\hline Sleep & $3.07 \pm 0.19$ & $15.98 \pm 5.07$ & $3.67 \pm 0.24$ & $11.94 \pm 3.48$ & $2.97 \pm 0.27$ & $9.77 \pm 2.96$ \\
Wake & $2.57 \pm 0.19$ & $5.31 \pm 1.11$ & $3.10 \pm 0.24$ & $5.53 \pm 1.38$ & $2.90 \pm 0.21$ & $9.49 \pm 2.93$ \\
\hline
\end{tabular}

Table 3. Scores from the Stanford Sleepiness Scale (SSS) and the proportion of attentional lapses made in the psychomotor vigilance task (PVT) for each session. Mean (SD).

\section{Psychomotor vigilance task}

Attentional lapses (reaction times exceeding 500 ms; Lim \& Dinges, 2008) on the psychomotor vigilance task (PVT; see Table 3) were subjected to a 2 (Group: Sleep/Wake) * 3 (Session: Encoding/12 h/24 h) mixed ANOVA. One participant was excluded from this analysis as no responses were recorded for one of their sessions. There were no main effects of Group $(F(1$, $57)=2.24, p=.143)$ or Session $(F(2,144)=0.50, p=.606)$. A Group * Session interaction was observed $\left(F(2,114)=3.75, p=.027, \eta_{p}^{2}=.06\right)$, with participants in the sleep group having more attentional lapses at encoding than participants in the wake group $(t(57)=2.02, p=$ $.048, d=.53)$. However, this between-group difference did not survive a Bonferroni-corrected alpha threshold of $p \leq .006$.

\section{Discussion}

We assessed the impact of rehearsal and prior knowledge on sleep-associated memory consolidation in a pre-registered, online study. In keeping with the previously reported benefits of sleep for offline memory processing, source memory retention was higher at the $12 \mathrm{~h}$ follow-up in the sleep group than the wake group (an advantage that had subsided by the $24 \mathrm{~h}$ follow-up once participants in the wake group had themselves had the opportunity for sleep). Although rehearsal strategy had no effect on the memory benefits of sleep at the $12 \mathrm{~h}$ follow-up, a significant interaction emerged at the $24 \mathrm{~h}$ follow-up, such that restudied memories were better remembered than retested memories, but only in the sleep group (i.e. those individuals who had slept soon after learning). Prior knowledge also influenced overnight memory processing, but in the opposite direction to that predicted by our hypothesis. The retention advantage for plausible (i.e. schematically congruent) relative to implausible (i.e. schematically incongruent) pairings was smaller in the sleep group than the wake group at the $12 \mathrm{~h}$ follow-up, and this effect was supported by a subsidiary analysis of the $24 \mathrm{~h}$ assessment. Hence, sleep appeared to preferentially strengthen memories that did not conform to participants' pre-existing, schematic knowledge.

That only the sleep group exhibited greater retrieval of restudied relative to retested memories at the $24 \mathrm{~h}$ follow-up is in keeping with earlier studies assessing the impact of rehearsal strategy on sleep-associated consolidation (Bäuml et al., 2014; Antony \& Paller, 2018). It has been suggested that retrieval practice provides a "fast route" to consolidation 
by rapidly strengthening newly acquired memories, potentially via similar mechanisms to those underpinning the memory benefits of sleep (Antony et al., 2017). Overnight memory processes may therefore bypass retested memories, prioritising the stabilisation of restudied, and proportionately unconsolidated, information.

Importantly, our findings extend those of previous work by demonstrating that the selective benefits of sleep for restudied information only emerge when sleep occurs soon after learning. Although prior studies have suggested that overnight consolidation effects are strongest for information learned within a few hours of sleep (Ashton \& Cairney, 2021; Gais, Lucas, \& Born, 2006; Payne, et al., 2012; Talamini et al., 2008), our current study shows that there might also be a time-critical relationship between memory rehearsal and sleepassociated memory gains. This has potentially important practical implications for learning and education (e.g. in terms of the timing of study sessions when preparing for an examination).

It should be noted, however, that if the benefits of sleep for restudied information are contingent on sleep taking place soon after learning, then one might have expected a sleepmemory effect to emerge for restudied relative to retested associations at both the $12 \mathrm{~h}$ and $24 \mathrm{~h}$ follow-ups (whereas only the latter was observed in the current study). A potential explanation for this discrepancy relates to overall performance levels at the $12 \mathrm{~h}$ follow-up, which were generally high, and may have prevented rehearsal strategy from influencing sleep-associated consolidation until the $24 \mathrm{~h}$ assessment. Indeed, previous work has shown that extensive memory training (such as that used in our encoding phase) can prevent rehearsal strategies from influencing performance until several days after training (Antony \& Paller, 2018). Hence, a certain degree of memory decay might have been necessary for a selective influence of sleep on restudied information to emerge in our data.

Our observation that memory retention was improved for schematically congruent (vs incongruent) associations is in keeping with a large body of work on the memory benefits of cognitive schemata (Cycowicz, Nessler, Horton, \& Friedman, 2008; Liu, Grady, \& Moscovitch, 2017). However, in direct contrast to our hypothesis, we found that sleep-associated memory gains were amplified for implausible (i.e. schematically incongruent) relative to plausible (i.e. schematically congruent) noun-colour pairings. We observed this effect in both the sleep and wake groups after isolating memory retention across their respective intervals of overnight sleep, suggesting that sleep may offer larger advantages to memories that deviate from our existing knowledge of the world.

This seemingly preferential impact of sleep on schematically incongruent memories appears to oppose theoretical frameworks arguing that sleep actively underpins both schema formation and the addition of new knowledge into existing schemata (Lewis \& Durrant, 2011). However, because schematically incongruent declarative associations are thought to be 
stored as episodic representations in medial temporal lobe (van Kesteren et al., 2012), and newly formed episodic memories are thought to be reactivated in hippocampus during postlearning sleep (Rasch \& Born, 2013), it is possible that these non-conformant, episodic memories are selectively strengthened in the sleeping brain. Relatedly, previous studies reporting enhanced overnight memory gains for schema-conformant information have used paradigms that differ considerably from the source memory task employed in the current study; for example, assessing memory for melodic sequences (Durrant et al., 2015) or route learning abilities (Wamsley et al., 2010). Delineating the specific conditions under which sleep supports the consolidation of schematically congruent or incongruent information is an important challenge for future research, and will enhance our understanding of the fundamental organisation of memory.

It is nevertheless important to consider that memory retention at baseline was generally poorer for implausible than plausible noun-colour pairings, suggesting that implausible associations were more weakly encoded than their plausible counterparts. Because previous work has shown that the memory benefits of sleep are more robust for weakly relative to strongly encoded materials (Denis et al., 2021, 2020; Payne, Chambers, \& Kensinger, 2012; Schmidt et al., 2006, but also see Petzka et al., 2021), the amplified overnight memory gains for implausible (vs plausible) memories observed in the current study might reflect an impact of memory strength, rather than schematic congruency, on sleep-associated consolidation. Yet, if this were the case, a three-way interaction should have emerged in our Group * Rehearsal Strategy * Plausibility ANOVA, with the weakest memories (i.e. non-rehearsed and implausible noun-colour pairings) showing the greatest enhancement across overnight sleep.

In conclusion, our online study adds to an extensive body of literature demonstrating that sleep supports offline memory processing (Ashton \& Cairney, 2021; Ashton et al., 2020; Cairney, Lindsay, Paller, \& Gaskell, 2018b; Durrant et al., 2016; Rasch \& Born, 2013). Furthermore, our data suggest that: 1 ) the memory benefits of sleep are enhanced for restudied information (as compared to information that has undergone retrieval practice), but only when sleep occurs soon after learning, and 2) sleep-associated memory gains are bolstered for schematically incongruent (relative to schematically congruent) associations. These findings provide new insights into the interaction of prior knowledge, online rehearsal strategies and offline consolidation processes.

\section{Acknowledgements}

This work was supported by a Medical Research Council Career Development Award (MR/P020208/1) to S.A.C. Study data can be retrieved via the following link: osf.io/vcfgm

\section{References}

Antony, J. W., Ferreira, C. S., Norman, K. A., \& Wimber, M. (2017). Retrieval as a Fast Route to Memory Consolidation. Trends in Cognitive Sciences, 21(8), 573-576. 
https://doi.org/10.1016/j.tics.2017.05.001

Antony, J. W., \& Paller, K. A. (2018). Retrieval and sleep both counteract the forgetting of spatial information. Learning \& Memory, 25(6), 258-263.

https://doi.org/10.1101//m.046268.117

Antony, J. W., Schönauer, M., Staresina, B. P., \& Cairney, S. A. (2019). Sleep Spindles and Memory Reprocessing. Trends in Neurosciences. https://doi.org/10.1016/j.tins.2018.09.012

Ashton, J. E., \& Cairney, S. A. (2021). Future-relevant memories are not selectively strengthened during sleep. PLOS ONE, In Press.

Ashton, J. E., Harrington, M. O., Langthorne, D., Ngo, H. V. V., \& Cairney, S. A. (2020). Sleep deprivation induces fragmented memory loss. Learning and Memory, 27(4), 130-135. https://doi.org/10.1101/Im.050757.119

Ashton, J. E., Jefferies, E., \& Gaskell, M. G. (2018). A role for consolidation in cross-modal category learning. Neuropsychologia, 108, 50-60.

https://doi.org/10.1016/j.neuropsychologia.2017.11.010

Bäuml, K. H. T., Holterman C., C., \& Abel M., M. (2014). Sleep can reduce the testing effect: It enhances recall of restudied items but can leave recall of retrieved items unaffected. Journal of Experimental Psychology: Learning Memory and Cognition. https://doi.org/10.1037/xlm0000025

Cairney, S. A., Lindsay, S., Paller, K. A., \& Gaskell, M. G. (2018a). Sleep preserves original and distorted memory traces. Cortex, 99, 39-44. https://doi.org/https://doi.org/10.1016/j.cortex.2017.10.005

Cairney, S. A., Lindsay, S., Sobczak, J. M., Paller, K. A., \& Gaskell, M. G. (2016). The Benefits of Targeted Memory Reactivation for Consolidation in Sleep are Contingent on Memory Accuracy and Direct Cue-Memory Associations. SLEEP. https://doi.org/10.5665/sleep.5772

Carpenter, S. K., Pashler, H., Wixted, J. T., \& Vul, E. (2008). The effects of tests on learning and forgetting. Memory \& Cognition, 36(2), 438-448.

https://doi.org/10.3758/MC.36.2.438

Creery, J. D., Oudiette, D., Antony, J. W., \& Paller, K. A. (2015). Targeted memory reactivation during sleep depends on prior learning. Sleep, 38(5), 755-763. https://doi.org/10.5665/sleep.4670

Cycowicz, Y. M., Nessler, D., Horton, C., \& Friedman, D. (2008). Retrieving object color: The influence of color congruity and test format. NeuroReport, 19(14), 1387-1390. https://doi.org/10.1097/WNR.0b013e32830c8df1

Denis, D., Mylonas, D., Poskanzer, C., Bursal, V., Payne, J. D., \& Stickgold, R. (2021). Sleep Spindles Preferentially Consolidate Weakly Encoded Memories. The Journal of Neuroscience, 41(18), 4088-4099. https://doi.org/10.1523/jneurosci.0818-20.2021

Denis, D., Schapiro, A. C., Poskanzer, C., Bursal, V., Charon, L., Morgan, A., \& Stickgold, R. (2020). The roles of item exposure and visualization success in the consolidation of memories across wake and sleep. Learning and Memory, 27(11), 451-456. https://doi.org/10.1101/LM.051383.120

Diekelmann, S., \& Born, J. (2010). The memory function of sleep. Nature Reviews Neuroscience, 11(2), 114-126. https://doi.org/10.1038/nrn2762

Durrant, S. J., Cairney, S. A., \& Lewis, P. A. (2016). Cross-modal transfer of statistical information benefits from sleep. Cortex, 78, 85-99.

https://doi.org/10.1016/j.cortex.2016.02.011 
Durrant, S. J., Cairney, S. A., McDermott, C., \& Lewis, P. A. (2015). Schema-conformant memories are preferentially consolidated during REM sleep. Neurobiology of Learning and Memory, 122, 41-50. https://doi.org/10.1016/j.nlm.2015.02.011

Gais, S., Lucas, B., \& Born, J. (2006). Sleep after learning aids memory recall. Learning \& Memory, 13(3), 259-262. https://doi.org/10.1101/Im.132106

Gaskell, M. G., Cairney, S. A., \& Rodd, J. M. (2019). Contextual priming of word meanings is stabilized over sleep. Cognition, 182, 109-126. https://doi.org/10.1016/j.cognition.2018.09.007

Groch, S., Schreiner, T., Rasch, B., Huber, R., \& Wilhelm, I. (2017). Prior knowledge is essential for the beneficial effect of targeted memory reactivation during sleep. Scientific Reports, 7, 39763. https://doi.org/10.1038/srep39763

Hoddes, E., Zarcone, V., Smythe, H., Phillips, R., \& Dement, W. C. (1973). Quantification of Sleepiness: A New Approach. Psychophysiology, 10(4), 431-436. https://doi.org/10.1111/j.1469-8986.1973.tb00801.x

Khitrov, M. Y., Laxminarayan, S., Thorsley, D., Ramakrishnan, S., Rajaraman, S., Wesensten, N. J., \& Reifman, J. (2014). PC-PVT: A platform for psychomotor vigilance task testing, analysis, and prediction. Behavior Research Methods, 46(1), 140-147. https://doi.org/10.3758/s13428-013-0339-9

Klinzing, J. G., Niethard, N., \& Born, J. (2019). Mechanisms of systems memory consolidation during sleep. Nature Neuroscience, 22(10), 1598-1610. https://doi.org/10.1038/s41593-019-0467-3

Lewis, P. A., \& Durrant, S. J. (2011). Overlapping memory replay during sleep builds cognitive schemata. Trends in Cognitive Sciences, 15(8), 343-351. https://doi.org/10.1016/j.tics.2011.06.004

Lim, J., \& Dinges, D. F. (2008). Sleep deprivation and vigilant attention. In Annals of the New York Academy of Sciences (Vol. 1129, pp. 305-322). https://doi.org/10.1196/annals.1417.002

Liu, Z. X., Grady, C., \& Moscovitch, M. (2017). Effects of prior-knowledge on brain activation and connectivity during associative memory encoding. Cerebral Cortex, 27(3), 19912009. https://doi.org/10.1093/cercor/bhw047

Lo, J. C., Dijk, D.-J. J., \& Groeger, J. A. (2014). Comparing the effects of nocturnal sleep and daytime napping on declarative memory consolidation. PloS One, 9(9), e108100. https://doi.org/10.1371/journal.pone.0108100

Payne, J. D., Chambers, A. M., \& Kensinger, E. A. (2012). Sleep promotes lasting changes in selective memory for emotional scenes. Frontiers in Integrative Neuroscience, 6, 108. https://doi.org/10.3389/fnint.2012.00108

Payne, J. D., Tucker, M. A., Ellenbogen, J. M., Wamsley, E. J., Walker, M. P., Schacter, D. L., \& Stickgold, R. (2012). Memory for Semantically Related and Unrelated Declarative Information: The Benefit of Sleep, the Cost of Wake. PLOS ONE, 7(3), 1-7. https://doi.org/10.1371/journal.pone.0033079

Peirce, J., Gray, J. R., Simpson, S., MacAskill, M., Höchenberger, R., Sogo, H., ... Lindeløv, J. K. (2019). PsychoPy2: Experiments in behavior made easy. Behavior Research Methods. https://doi.org/10.3758/s13428-018-01193-y

Petzka, M., Charest, I., Balanos, G. M., \& Staresina, B. P. (2021). Does sleep-dependent consolidation favour weak memories? Cortex, 134, 65-75. https://doi.org/10.1016/j.cortex.2020.10.005

R Core Team. (2019). R: A language and environment for statistical computing. $R$ Foundation 


\section{for Statistical Computing.}

Rasch, B., \& Born, J. (2007). Maintaining memories by reactivation. Current Opinion in Neurobiology, 17(6), 698-703. https://doi.org/10.1016/j.conb.2007.11.007

Rasch, B., \& Born, J. (2013). About sleep's role in memory. Physiological Reviews, 93(2), 681-766. https://doi.org/10.1152/physrev.00032.2012

Roediger, H. L., \& Karpicke, J. D. (2006). Test-Enhanced Learning. Psychological Science, 17(3), 249-255. https://doi.org/10.1111/j.1467-9280.2006.01693.x

Schechtman, E., Lampe, A., Wilson, B. J., Kwon, E., Anderson, M. C., \& Paller, K. A. (2021). Sleep reactivation did not boost suppression-induced forgetting. Scientific Reports, 11(1). https://doi.org/10.1038/s41598-020-80671-w

Schmidt, C., Peigneux, P., Muto, V., Schenkel, M., Knoblauch, V., Münch, M., ... Cajochen, C. (2006). Encoding difficulty promotes postlearning changes in sleep spindle activity during napping. Journal of Neuroscience, 26(35), 8976-8982.

https://doi.org/10.1523/JNEUROSCI.2464-06.2006

Sommer, T. (2017). The emergence of knowledge and how it supports the memory for novel related information. Cerebral Cortex, 27(3), 1906-1921. https://doi.org/10.1093/cercor/bhw031

Staresina, B. P., \& Davachi, L. (2008). Selective and shared contributions of the hippocampus and perirhinal cortex to episodic item and associative encoding. Journal of Cognitive Neuroscience, 20(8), 1478-1489. https://doi.org/10.1162/jocn.2008.20104

Talamini, L. M., Nieuwenhuis, I. L. C., Takashima, A., \& Jensen, O. (2008). Sleep directly following learning benefits consolidation of spatial associative memory. Learning \& Memory, 15(4), 233-237. https://doi.org/10.1101//m.771608

Tse, D., Langston, R. F., Kakeyama, M., Bethus, I., Spooner, P. A., Wood, E. R., ... Morris, R. G. M. (2007). Schemas and Memory Consolidation. Science, 316(5821), 76-82. https://doi.org/10.1126/science.1135935

Tse, D., Takeuchi, T., Kakeyama, M., Kajii, Y., Okuno, H., Tohyama, C., ... Morris, R. G. M. (2011). Schema-dependent gene activation and memory encoding in neocortex. Science, 333(6044), 891-895. https://doi.org/10.1126/science.1205274

van Kesteren, M. T. R. R., Beul, S. F., Takashima, A., Henson, R. N., Ruiter, D. J., \& Fernández, G. (2013). Differential roles for medial prefrontal and medial temporal cortices in schema-dependent encoding: From congruent to incongruent. Neuropsychologia, 51(12), 2352-2359. https://doi.org/10.1016/j.neuropsychologia.2013.05.027

van Kesteren, M. T. R., Ruiter, D. J., Fernández, G., \& Henson, R. N. (2012). How schema and novelty augment memory formation. Trends in Neurosciences. https://doi.org/10.1016/j.tins.2012.02.001

Wamsley, E. J., Tucker, M. A., Payne, J. D., \& Stickgold, R. (2010). A brief nap is beneficial for human route-learning: The role of navigation experience and EEG spectral power. Learning and Memory. https://doi.org/10.1101//m.1828310 